\title{
A APROXIMAÇÃO DE TRABALHOS ACADÊMICOS NO UNIVERSO ORGANIZACIONAL NAS CIÊNCIAS ADMINISTRATIVAS: UMA ANÁLISE DOS TRABALHOS DO ENCONTRO DA ASSOCIAÇÃO NACIONAL DE PÓS- GRADUAÇÃO E PESQUISA EM ADMINISTRAÇÃO DE 2014
}

\author{
Nelson Guilherme Machado Pinto ${ }^{1}$, Bruno Pereira Conte ${ }^{2}$, Rodrigo Abbade da Silva ${ }^{3}$, \\ Daniel Arruda Coronel ${ }^{4}$
}

\section{RESUMO}

O objetivo deste trabalho consistiu em analisar os artigos apresentados no Encontro da Associação Nacional de Pós-Graduação e Pesquisa em Administração (EnANPAD), em 2014, a fim de verificar se os trabalhos deste evento da área de Administração possuem uma aproximação com universo organizacional. De maneira geral, os trabalhos demonstraram certa aproximação com universo organizacional, com diferenças significativas entre as áreas temáticas, carecendo de uma maior aproximação com empresas visando à realização de estudos e ao aprimoramento das técnicas de análises estudadas, a fim de ajustar, de forma mais acentuada, a teoria e a prática. Esse deve ser o caminho para que o mais importante evento de Administração do Brasil apresente em edições futuras, um maior nível de orientação à prática organizacional.

Palavras-chave: Trabalhos Acadêmicos; Universo Organizacional; Administração.

\section{THE APPROACH OF ACADEMIC WORKS IN THE ORGANIZATIONAL ENVIRONMENT IN ADMINISTRATIVE SCIENCES: AN ANALYSIS OF THE STUDIES AT THE NATIONAL ASSOCIATION OF GRADUATE STUDIES AND RESEARCH IN ADMINISTRATION OF 2014}

\begin{abstract}
The objective of this study was to analyze the articles submitted to the National Association of Graduate Studies and Research in Administration (EnANPAD) in 2014 in order to verify if the studies related to Administration presented at the event have an approximation to the organizational universe. Overall, the studies showed some approximation to the organizational environment, with significant differences between the thematic areas, lacking a greater approach regarding the organizations with the objective to conduct studies and improve the techniques of analysis studied, in order to bring closer the theory and practice. Which would enable the most important event of Administration in Brazil to present a higher level of guidance to organizational practice in future editions.
\end{abstract}

Keywords: Academic Studies; Organizational Universe; Administration.

\section{INTRODUÇÃO}

A educação é uma das principais ferramentas de desenvolvimento e agente de mudanças em uma sociedade. O processo de educação é iniciado dentro de um contexto familiar, contudo ele é amplamente debatido dentro das escolas e universidades. Nessas

\footnotetext{
${ }^{1}$ Universidade Federal de Santa Maria

${ }^{2}$ Universidade Federal de Santa Maria

${ }^{3}$ Universidade Federal de Santa Maria

${ }^{4}$ Universidade Federal de Santa Maria 
últimas instituições, responsáveis pela formação do jovem profissional que estará atuando no mercado, uma das grandes discussões se refere ao questionamento se o aprendizado em sala de aula é aplicável em situações da realidade fora do contexto das instituições de ensino.

Os debates entre o relacionamento de teóricos e práticos acabam levando a alguns conflitos entre os envolvidos. Isso porque os práticos acusam os teóricos de realizarem estudos sem resultados que levem de fato a resoluções na realidade, enquanto que os teóricos acusam os práticos de comportamentos superficiais e sem o entendimento de diversos aspectos. Diante desse contexto, uma das soluções para essa situação crítica, é a junção do conhecimento científico de forma conjunta com situações práticas e experimentais (ALBACH, 1993; DIJK; PUNCH, 1993).

Essa discussão acaba acentuando-se dentro do contexto dos centros de ensino em Administração. Isso porque os estudantes dessa ciência questionam a cientificidade da mesma bem como se há uma relação entre teoria e prática (ALBACH, 1993). Ademais, essa situação é fomentada dentro da área de Administração por dois aspectos, conforme afirmam Johann e Duclós (2013). O primeiro é que muitos alunos buscam situações que remontem a aspectos da realidade a fim de não ficarem apenas na parte teórica, e o segundo aspecto é que os professores têm conhecimento de que, em alguns casos, muito do que é ensinado em sala de aula não será utilizado em situações futuras pelos alunos por não ser aplicável de fato à realidade organizacional.

Diante desse contexto, existem muitos questionamentos para o fato de reconhecer se os esforços teóricos da área de Administração levam a alguma contribuição para a prática organizacional. Dessa forma, as pesquisas organizacionais acabam adquirindo um caráter abstrato, não se aproximando do universo das organizações. Isso é consequência do distanciamento real e metodológico que existe entre o indivíduo que realiza o estudo e seu objeto de análise (AUDET, 1986).

A orientação da teoria da Administração para a prática organizacional pode ser definida pela capacidade de compreender e dominar os problemas da empresa, descobrindo-os e formulando soluções. Para a ocorrência dessa orientação, são necessários os conhecimentos, as capacidades e as habilidades na teoria administrativa de um indivíduo ligado à empresa (ALBACH, 1993). Dessa forma, os estudos organizacionais, além de um componente e contribuição teóricos, devem levar em consideração os aspectos da prática organizacional a fim de trazerem avanços e resultados para auxiliar os praticantes na realização de suas atividades diárias dentro do contexto das organizações (DJALÓ; PROCOPIUCK, 2010). 
A partir disso, a pesquisa em Administração busca a verdade a qual reúne, analisa, interpreta e relata informações para que as decisões e questões administrativas se tornem mais eficazes. Assim, as pesquisas em Administração procuram por meio de aspectos teóricos relacionarem-se a aspectos práticos para que as melhores decisões sejam tomadas diante de um contexto organizacional (HAIR et al., 2005).

Neste sentido, o objetivo deste trabalho consistiu em analisar os artigos apresentados no Encontro da Associação Nacional de Pós-Graduação e Pesquisa em Administração (EnANPAD), em 2014, a fim de verificar se os trabalhos deste evento da área de Administração possuem uma aproximação com o universo organizacional. Para o atendimento desse objetivo, foi utilizado um roteiro de análise, baseado nos modelos de Albach (1993) e Johann e Duclós (2013), para verificar se os trabalhos selecionados possuem as características que confirmam ou não essa orientação para o contexto das organizações. A escolha do EnANPAD é motivada por este ser um evento de referência para a área de Administração no Brasil, quando os pesquisadores da área no país reúnem-se anualmente para discutir diversos pontos e tendências da área.

A fim de alcançar seu objetivo, o presente estudo está estruturado em quatro seções, além desta introdução. Na segunda, é apresentado o referencial teórico; na seção seguinte, descrevem-se os procedimentos metodológicos utilizados; na quarta seção, os resultados são analisados e discutidos e, por fim, apresentam-se as principais considerações finais do trabalho.

\section{REFERENCIAL TEÓRICO}

A cientificidade da Administração pode ser um aspecto no qual alguns questionamentos podem emergir. Isso porque, conforme afirma Albach (1993), muitos executivos que exercem altos cargos administrativos ignoram, no dia a dia da condução de seus negócios, as teorias administrativas existentes. $\mathrm{O}$ tratamento da Administração como ciência passa a ser questionado a tal ponto de não se considerar essa área como uma ciência. Um exemplo disso é o Centro Nacional de Pesquisa da França, o qual não reconhece a Administração como ciência, mas, sim, como a aplicação de forma conjunta de muitas ciências que são consideradas básicas, tais como a Economia, a Matemática, a Psicologia e a Sociologia (JOHANN; DUCLÓS, 2013).

É diante desse debate sobre a cientificidade ou não da Administração que a aproximação de trabalhos acadêmicos com a prática organizacional passa a existir. Isso 
porque, de acordo com Rodrigues (2013), a ciência só tem prestígio na medida em que serve de base para as questões práticas. Dessa forma, de pouco adianta para a área empresarial um trabalho teórico se ele não tiver utilidade para situações que remontam e sejam aplicadas à realidade.

Assim, o relacionamento entre aspectos teóricos e práticos é o que garante que o caráter científico na prática organizacional proporcione avanços na parte teórica bem como nos aspectos das diversas áreas da empresa. Isso porque a ciência com orientação para a prática não possui um fim em si mesmo, visto que estará aberta à inovação e ao progresso. A fim de atender aos aspectos práticos, a teoria não pode ser uma "ciência pura", isto é, totalmente livre de qualquer necessidade e relacionamento com o contexto social. Entretanto, a teoria deve possuir suas autonomias, não se comportando como uma "ciência escrava", pois não deve estar sujeita a todas as demandas político-econômicas da sociedade e precisa ter certo grau de liberdade (BOURDIEU, 2004).

Apesar dos conflitos entre os aspectos teóricos e práticos dentro do contexto de estudos em Administração e da importância que a teoria deve dar à prática para ser aplicável, é válido destacar que os práticos sentem necessidade de educação de forma mais teórica para o aperfeiçoamento de suas atividades diárias. Dessa forma, os gestores buscam a educação gerencial por meio de cursos rápidos de gestão, os quais, na maioria das vezes, são ministrados por outros gestores e não por cientistas com um conhecimento mais teórico sobre a área. Diante dessa situação, o aprendizado efetivo da gestão não é atingido (DIJK; PUNCH, 1993).

Apesar disso, é preciso que a Administração, tanto como ciência quanto como prática gerencial tenha o alinhamento entre teoria e prática para almejar sucesso nesses dois âmbitos. Um exemplo desse alinhamento dentro do contexto organizacional são as universidades americanas. As empresas dos Estados Unidos acabam oportunizando aos professores das universidades o uso de seus casos em suas atividades de ensino e pesquisa (JOHANN; DUCLÓS, 2013).

Além disso, o conflito entre teóricos e práticos na Administração é originado pela existência de alguns conflitos dentro do campo teórico da área, principalmente quanto aos paradigmas adotados. Esses, na visão de Kuhn (1991), são um conjunto de crenças, valores e técnicas compartilhadas entre os membros de determinada comunidade científica que são aceitos pela grande maioria dos participantes, tornando-se referência para as abordagens de pesquisa dentro dessa área de conhecimento. 
Tendo isso em mente, dentro da Administração os principais paradigmas conflitantes são o positivismo e o interpretativismo. Enquanto o positivismo resgata conceitos das Ciências Naturais e Exatas, levantando aspectos principalmente quantitativos e objetivos para o contexto organizacional, o paradigma interpretativista posiciona-se como uma crítica ao primeiro, levando em consideração aspectos mais subjetivos dentro do campo da área. Contudo, os conflitos científicos dentro da Administração não se restringem apenas a argumentos epistemológicos e intelectuais, passando a envolver questões de grupos de interesse, reconhecimento e prestígio. Dessa forma, alguns pontos fundamentais dentro da pesquisa científica da área são esquecidos, tais como a qualidade, a consistência, o rigor e a relevância de um trabalho (BARBOSA et al., 2013).

Considerando-se essa realidade, grande parte dos pesquisadores em Administração pode ser dividida em dois grupos que fomentam ainda mais essa rivalidade entre aspectos teóricos e práticos. O primeiro grupo é formado pelos pesquisadores que se consideram teóricos, isto é, estudam a parte histórica de como a teoria é utilizada nas estruturas organizacionais e como é manifestada na interação com os atores organizacionais. De outro lado, está o grupo de pesquisadores que se classifica como praticante das técnicas gerenciais, que também levam em consideração a história, mas com uma preocupação na forma pela qual esta contribui na objetividade da organização (DJALÓ; PROCOPIUCK, 2010).

Por último, é válido destacar que a produção acadêmica em Administração, no Brasil e no mundo, está em constante crescimento. Isso pode ser corroborado pelo acréscimo no número de estudos submetidos e publicados em eventos e periódicos da área, além do aumento de dissertações e teses em Administração nos últimos anos. Contudo, esse crescimento quantitativo não é acompanhado por questões qualitativas (BERTERO, CALDAS e WOOD JR, 1999). Além disso, apesar de toda a discussão entre aspectos teóricos e práticos dentro da Administração para o rumo futuro da área, verifica-se a disposição de poucos estudiosos com o objetivo de mudar a forma e as estruturas que sustentam o paradigma atual das pesquisas em Administração (WEICK, 1992).

\section{PROCEDIMENTOS METODOLÓGICOS}

A presente pesquisa caracteriza-se pelo seu aspecto exploratório, visto que procura estabelecer uma maior familiaridade e percepção com um determinado tema (GIL, 2010). Ademais, destaca-se o caráter aplicado do trabalho, pois a sua natureza é elaborada a fim de adquirir conhecimentos para aplicação em um tema específico (MARCONI; LAKATUS, 
2005). Dessa forma, o estudo procurou fomentar a discussão entre a aproximação de trabalhos acadêmicos ao contexto do universo organizacional dentro da área das Ciências Administrativas.

Além disso, o presente estudo utilizou-se de uma técnica indireta de tratamento de dados, pois, a partir do levantamento bibliográfico, foram elaboradas as análises do estudo. No que se refere ao procedimento metodológico, utilizou-se o método monográfico e comparativo (MARCONI; LAKATUS, 2005). Portanto, foram utilizados, como objetos de estudo, 737 artigos científicos dos 880 apresentados e publicados no Encontro da Associação Nacional de Pós-Graduação e Pesquisa em Administração do Ano de 2014. A redução de 143 artigos deve-se ao fato de estes serem apresentados na forma de resumo inviabilizando a análise, sendo utilizados, portanto, os 737 artigos publicados de forma completa nos anais no evento.

A escolha do referido evento para constatação da aproximação dos estudos ao contexto organizacional, deve-se ao fato de o EnANPAD ser um evento referência no Brasil para estudos em Administração. A fim de avaliar a orientação à prática dos trabalhos do EnANPAD 2014, utilizou-se como instrumento para verificação desse fato um roteiro de análise baseado nos modelos de Albach (1993) e Johann e Duclós (2013). A partir desses dois trabalhos foram levantados os seguintes questionamentos a fim de verificar a aproximação dos trabalhos ao universo organizacional:

a) o estudo utiliza-se da técnica de estudo de caso;

b) a transparência teórica do estudo melhorou a prática organizacional;

c) existe disponibilidade para diálogo entre teóricos e práticos;

d) é possível formular enunciados do tipo "se, então", denotando relações de causalidade na formulação dos problemas da pesquisa;

e) não existência de juízos de valor na elaboração do estudo;

f) os enunciados científicos do trabalho possuem objetividade, isto é, permitem a verificação e aplicação para elaboração de trabalhos de terceiros; e

g) os enunciados científicos do trabalho são falseáveis, ou seja, permite a elaboração de transformações a fim de melhorá-los.

A utilização do estudo de caso como orientação à prática dos estudos organizacionais é justificada por essa técnica apresentar forte ligação da academia com a realidade do mundo 
organizacional atual. O segundo item de avaliação é utilizado para verificar se os apontamentos teóricos do estudo obtiveram alguma contribuição e avanço no dia a dia das organizações. Corroborando esse quesito, é pertinente verificar se, além dessa melhora nas práticas organizacionais, o estudo foi capaz de fazer um diálogo entre teóricos e práticos visando à aproximação entre esses dois agentes que estão em constante conflito conforme aponta a literatura acadêmica (JOHANN; DUCLÓS, 2013).

A questão da causalidade refere-se às relações tanto teóricas como práticas que o estudo é capaz de formular a fim de obter aproximação desses dois aspectos. O aspecto relacionado aos juízos de valores relaciona-se ao fato de que o estudo deva vir a contribuir para a prática organizacional com a formulação, a análise e o julgamento baseado em questões teóricas e não em aspectos ligado ao senso comum. Por fim, os dois últimos critérios referemse à reprodutibilidade para que os estudos da área possam continuar avançando e a elaboração de transformações com o objetivo de melhorar os trabalhos baseados na falseabilidade de Karl Popper $^{5}$, respectivamente (ALBACH, 1993).

As análises foram realizadas de forma individual com base na leitura hermenêutica de cada artigo científico. Para os sete questionamentos, foi estabelecida uma escala nominal binária, sim/não, podendo o artigo apresentar as características (SIM) ou não apresentá-las (NÃO), conforme procedimento utilizado por Johann e Duclós (2013). A fim de verificar a evolução da orientação a prática dos artigos do EnANPAD 2014, os mesmos foram divididos nas onze áreas temáticas do evento, que são:

a) ADI - Administração da Informação;

b) APB - Administração Pública;

c) $\mathrm{CON}$ - Contabilidade;

d) EOR - Estudos Organizacionais;

e) EPQ - Ensino e Pesquisa em Administração e Contabilidade;

f) ESO - Estratégia em Organizações;

g) FIN - Finanças;

h) GCT - Gestão da Ciência, Tecnologia e Inovação;

\footnotetext{
${ }^{5}$ Segundo Popper (1975), não existem garantias de que as observações passadas serão reproduzidas no futuro. A partir disso, considerando a ciência como um conjunto de hipóteses que podem ser propostas com a finalidade de detalhar ou explicar fenômenos do mundo, a falseabilidade de Popper (1975) baseia-se na possibilidade de testar essas hipóteses. Assim, na visão deste autor, para considerar uma hipótese como científica, deve haver a possibilidade de falsear (refutar) essas hipóteses a fim de dar continuidade à cientificidade de uma determinada área. 
i) GOL - Gestão de Operações e Logística;

j) GPR - Gestão de Pessoas e Relações de Trabalho;

k) MKT - Marketing.

A partir dos sete questionamentos em escala binária do estudo foi criada uma escala de sete pontos, pela qual os trabalhos com todas as respostas "sim" possuem pela metodologia de estudo aproximação do universo organizacional e uma escala de sete pontos, e os trabalhos com todas as respostas "não" possuem, pela metodologia de estudo, nenhuma aproximação do universo organizacional com uma escala de zero ponto. Diante desses dois extremos, de zero a sete, outras seis situações de pontuação na escala poderiam ser encontradas. A partir dessa escala, além das análises descritivas, testes de diferença de média, em função da normalidade ou não dos dados, foram realizados a fim de verificar as diferenças estatísticas dessa escala para as áreas temáticas pesquisadas.

É válido destacar que os trabalhos foram avaliados a partir dos questionamentos anteriores por meio do sistema de revisão dupla, também conhecido como double blind review. Portanto, cada trabalho foi avaliado por pelo menos dois autores deste estudo e, em caso de discordância em alguns dos pontos do trabalho, um terceiro autor foi solicitado a fim de tomar uma decisão final.

\section{Análise e Discussão dos Resultados}

Após a avaliação, por ao menos dois avaliadores, dos sete questionamentos para verificar a aproximação do universo organizacional dos estudos em Administração dos 737 artigos dos anais do EnANPAD 2014, os artigos foram agrupados pelas áreas temáticas do evento a fim de verificar o comportamento dos questionamentos nas diferentes áreas de estudo da Administração, conforme Tabela 1. 
Tabela 1 - Percentual (\%) de trabalhos do EnANPAD 2014 que possuíam as características conforme sua área temática

\begin{tabular}{cccccccc}
\hline Área & $\begin{array}{c}\text { Estudo } \\
\text { de caso }\end{array}$ & $\begin{array}{c}\text { Transparência } \\
\text { teórica melhorou } \\
\text { a prática }\end{array}$ & $\begin{array}{c}\text { Disponibilidade } \\
\text { para diálogo } \\
\text { teóricos/práticos }\end{array}$ & $\begin{array}{c}\text { Enunciados } \\
\text { do tipo "se, } \\
\text { então" }\end{array}$ & $\begin{array}{c}\text { Não } \\
\text { haver } \\
\text { juízos de } \\
\text { valor }\end{array}$ & $\begin{array}{c}\text { Enunciados } \\
\text { científicos } \\
\text { objetivos }\end{array}$ & $\begin{array}{c}\text { Enunciado } \\
\text { falseável }\end{array}$ \\
\hline ADI & 19,64 & 83,93 & 100,00 & 44,64 & 33,93 & 83,93 & 89,29 \\
APB & 29,82 & 98,25 & 100,00 & 44,74 & 30,70 & 92,98 & 89,47 \\
CON & 4,17 & 100,00 & 100,00 & 41,67 & 31,25 & 95,83 & 89,58 \\
EQR & 43,82 & 60,67 & 59,55 & 12,36 & 39,33 & 71,91 & 87,64 \\
EPQ & 47,46 & 32,20 & 22,03 & 15,25 & 23,73 & 76,27 & 88,14 \\
ESO & 41,12 & 45,79 & 39,25 & 39,25 & 52,34 & 71,03 & 88,79 \\
FIN & 8,11 & 40,54 & 59,46 & 91,89 & 45,95 & 94,59 & 89,19 \\
GCT & 34,78 & 63,04 & 76,09 & 32,61 & 80,43 & 84,78 & 93,48 \\
GOL & 33,33 & 79,49 & 79,49 & 41,03 & 71,79 & 92,31 & 100,00 \\
GPR & 33,33 & 57,69 & 69,23 & 34,62 & 58,97 & 87,18 & 100,00 \\
MKT & 11,29 & 61,29 & 77,42 & 59,68 & 85,48 & 96,77 & 100,00 \\
\hline Total & 30,34 & 66,26 & 70,20 & 26,80 & 41,50 & 84,63 & 91,84 \\
\hline Fonte: Elaborado pelos autores com base nos dados da pesquisa. & & & & &
\end{tabular}

As menores médias e as maiores diferenças entre as áreas dos artigos estudados referem-se aos aspectos de estudo de caso, ao efeito da transparência teórica na melhora da prática, aos enunciados do tipo "se, então" e à ausência de juízos de valor. De forma contrária a esses aspectos, os itens que verificaram enunciados objetivos e hipóteses falseáveis apresentaram maiores médias e menores oscilações entre as áreas temáticas. Enunciados objetivos referem-se aos estudos serem verificados e aplicados para elaboração de trabalhos de terceiros (ALBACH, 1993). Assim, nota-se o esforço de todas as áreas temáticas a fim de permitirem que os temas propostos e os resultados possam ser reproduzidos por outros estudiosos para que sejam verificadas semelhanças e diferenças com os trabalhos originais.

O segundo aspecto verificado que possui presença consensual nos trabalhos pesquisados está relacionado ao fato de os mesmos reproduzirem aspectos que podem ser falseáveis. Assim, os aspectos discutidos podem vir a ser refutados para que suas hipóteses sejam superadas e se dê continuidade à cientificidade da área (POPPER, 1975). Portanto, esses dois aspectos, com 100\% de presença nas áreas de Gestão de Operações e Logística, Gestão de Pessoas e Relações de Trabalho e Marketing e elevadas médias nas demais áreas temáticas do EnANPAD 2014, demonstram a preocupação dos trabalhos com a continuidade das discussões levantadas nos estudos para que eles possam ser expandidos e discutidos por outros pesquisadores a fim de contribuir para os avanços da área de Administração. 
O item de disponibilidade para diálogo entre teóricos e práticos foi, após os dois itens anteriores, com maiores frequências, o que mais esteve presente nos trabalhos observados. Assim, nota-se, durante a formulação dos trabalhos do EnANPAD, a aproximação entre esses dois grupos conflitantes. Isso de certa forma representa uma das saídas apresentadas para a diminuição dos conflitos desses dois grupos, o que deve ocorrer por meio da junção do conhecimento científico com situações práticas (DIJK e PUNCH, 1993). A exceção à alta frequências desses itens, com áreas com $100 \%$ de frequência nesse item, como por exemplo, Administração da Informação, Administração Pública e Contabilidade, foram as áreas de Ensino e Pesquisa em Administração e Contabilidade e Estratégia em Organizações. A primeira dessas áreas lida com questões mais voltadas a aspectos teóricos ligados ao ensino e à pesquisa da área, por isso o baixo valor para esse item, já a segunda dessas áreas possui preocupações mais ligadas a discussões práticas de muitos conceitos estratégicos da organização, sem se preocupar tanto ao relacionamento com aspectos teóricos.

O item que versava a respeito dos apontamentos teóricos do estudo que obtiverem alguma contribuição e avanço no dia a dia das organizações apresentou uma baixa frequência nas áreas de Ensino e Pesquisa em Administração e Contabilidade e Estratégia em Organizações. A primeira área, corroborando o que foi descrito anteriormente, acaba verificando aspectos que ficam com uma maior relevância para aspectos teóricos e acadêmicos do que para aspectos práticos. Já a área de Estratégia em Organizações acaba levantando aspectos, em alguns trabalhos, que demonstram apenas uma preocupação em descrever pontos estratégicos da organização, sem direcionar os estudos para contribuições possíveis que poderiam ser levantadas no contexto organizacional.

$\mathrm{O}$ aspecto analisado que apresentou as menores médias percentuais foi a questão do estudo de caso. Por essa ser uma técnica que apresenta forte ligação da academia com a realidade do mundo atual, percebe-se, em algumas áreas, um maior distanciamento entre esses dois pontos (JOHANN; DUCLÓS, 2013). Isso porque, as áreas de Contabilidade, Finanças e Marketing apresentam valores muito abaixo dos demais para esse quesito. Umas das justificativas para a não realização de estudos de caso nessas três áreas em detrimento das demais é a dificuldade, para o campo de Administração do Brasil, em verificar aspectos financeiros, contábeis e de características relevantes de mercado concorrencial e consumidor das empresas, os quais muitas vezes são sigilosos e apenas restritos a funcionários, demonstrando dificuldade para indivíduos (pesquisadores) externos à organização. Assim, fica explícito que, para a realidade estudada, tem-se um contexto onde os trabalhos 
acadêmicos mostram-se distantes do universo organizacional no que concerne às técnicas de análise, diferente do encontrado, por exemplo, nos Estados Unidos, conforme afirmam Johann e Duclós (2013), onde as empresas costumam oportunizar aos professores das universidades o uso de seus casos em suas atividades de ensino e pesquisa.

A presença da causalidade com questões do tipo "se, então" apenas demonstra a grande presença de estudos empíricos voltados a testar hipóteses e correlações causais dentro de algumas áreas de Administração, fato que tem sua origem na força do paradigma positivista dentro dessa área no Brasil (VIZEU, 2010). Dessa forma, as áreas de Finanças e Marketing destoam das demais, visto que relacionam os mais diversos aspectos com o objetivo de testar hipóteses, teorias e aspectos práticos, principalmente com a utilização de métodos quantitativos. Por último, verifica-se que a maioria dos trabalhos se utilizou de juízos de valor a fim de formular os enunciados e proposições dos estudos, visto que muitos trabalhos partem de aspectos práticos e do senso comum sem de fato possuírem justificativas teóricas para seus estudos.

A fim de detalhar um pouco mais a aproximação entre a prática organizacional de acordo com as áreas temáticas e o contexto organizacional dos 737 trabalhos do EnANPAD, eles foram analisados a partir de um índice, conforme Tabela 2.

Tabela 2 - Valor médio do índice de aproximação a prática organizacional dos trabalhos do EnANPAD 2014 que possuíam as características conforme sua área temática

\begin{tabular}{lc}
\hline Área & Valor médio do índice \\
\hline ADI & 4,55 \\
APB & 4,86 \\
CON & 4,63 \\
EQR & 3,75 \\
EPQ & 3,05 \\
ESO & 3,78 \\
FIN & 4,30 \\
GCT & 4,65 \\
GOL & 4,97 \\
GPR & 4,41 \\
MKT & 4,92 \\
Total & 4,31 \\
\hline
\end{tabular}

Fonte: Elaborado pelos autores com base nos dados da pesquisa.

Por meio de uma análise descritiva, verifica-se que, em sua média, os trabalhos do EnANPAD estão com certa orientação à prática organizacional, visto que a média do índice geral é de 4,31, isto é, maior que o valor do índice médio. Quanto às áreas temáticas 
verificam-se que a única área com valor abaixo do índice médio da escola de 3,5 é a área de Ensino e Pesquisa em Administração e Contabilidade com um valor de 3,05. Essa área, conforme análises realizadas anteriormente, tem sua maior preocupação com aspectos teóricos com um viés maior em melhorias no contexto de ensino e pesquisa da área do que com melhorias de fato na prática organizacional. Já as áreas com maiores valores de índice são a área de Gestão de Operações e Logística, com 4,97 de valor para o índice, e a área de Marketing com um valor de 4,92. Ambas as áreas possuem viés para operações práticas e de mercado, sendo processos gerenciais consolidados na ambiente empresarial (PEREIRA; TOLEDO; TOLEDO, 2009). Assim, justificam-se os maiores valores do índice de aproximação da prática organizacional do que as demais áreas.

A fim de verificar, as diferenças entre as áreas não apenas de forma descritiva, mas de forma estatística, um teste de diferença de médias foi realizado. Mas, para isso, foi preciso saber o pressuposto de normalidade dos dados. Neste sentido, foi realizado o Teste de Normalidade de Kolmogorov-Smirnov, que apresentou significância estatística ao nível de $1 \%$. Por isso, rejeita-se a hipótese nula do teste, que afirma que há normalidade entre os dados da formação do índice, devendo ocorrer, portanto, a utilização de testes não paramétricos, que, no caso desse estudo, é o teste de Kruskal-Wallis, conforme Tabela 3.

Tabela 3 - Teste de Kruskal-Wallis do índice de aproximação à prática organizacional dos trabalhos do EnANPAD 2014

\begin{tabular}{lccc}
\hline Variável & Qui-quadrado & Graus de Liberdade & Significância Estatística \\
\hline $\begin{array}{l}\text { Índice de aproximação } \\
\text { a prática organizacional }\end{array}$ & 145,17 & 10 & 0,001 \\
\hline \multicolumn{2}{l}{ Fonte: Elaborado pelos autores com base nos dados da pesquisa. }
\end{tabular}

O teste de Kruskal-Wallis apresenta significância estatística indicando que existe diferença da variável estudada nos seus grupos, ou seja, do índice de aproximação à prática organizacional nas áreas temáticas dos trabalhos do EnANPAD 2014. Os testes não paramétricos, diferentes dos testes paramétricos, não indicam o quanto um grupo é maior que o outro para uma variável. Entretanto, os testes não paramétricos indicam um ranking demonstrando qual variável é maior que a outra nos seus diferentes grupos. Assim, a Tabela 4 apresenta um ranking de posições para cada uma das áreas temáticas. 
Tabela 4 - Ranking do índice de aproximação a prática organizacional dos trabalhos do EnANPAD 2014 conforme sua área temática

\begin{tabular}{lc}
\hline Área & Ranking Médio \\
\hline ADI & 401,48 \\
APB & 454,25 \\
CON & 418,64 \\
EQR & 282,40 \\
EPQ & 169,03 \\
ESO & 276,97 \\
FIN & 374,04 \\
GCT & 430,74 \\
GOL & 479,29 \\
GPR & 384,31 \\
MKT & 468,60 \\
\hline
\end{tabular}

Fonte: Elaborado pelos autores com base nos dados da pesquisa.

Os resultados da Tabela 4 corroboram estatisticamente os resultados encontrados na análise descritiva. Isso porque as variáveis apresentam as mesmas colocações com a área de Ensino e Pesquisa em Administração e Contabilidade com a última colocação para o valor do índice e com a área de Gestão de Operações e Logística com a primeira colocação do valor do índice. De forma geral, os trabalhos do EnANPAD 2014 tentaram apresentar, em seus aspectos teóricos levantados, uma aproximação ao universo das organizações. Dificilmente haverá uma orientação e um relacionamento desses aspectos de forma total e mútua. Contudo, alguns pontos, como uma maior aproximação com as empresas para a realização de estudos e o aprimoramento das técnicas de análises estudadas, a fim de aproximar, de forma mais acentuada teoria e prática devem ser os caminhos para que o mais importante evento de Administração do Brasil apresente, em suas edições futuras, um maior nível de orientação à prática organizacional.

\section{CONSIDERAÇÕES FINAIS}

As discussões referentes a aspectos teóricos e práticos denotam um espaço de debate nas mais diversas áreas de conhecimento. Dentro da área de Administração não é diferente e é a partir desse conflito que a aproximação de trabalhos acadêmicos com relação ao universo organizacional na Administração foi discutida neste estudo. Verifica-se que o debate entre aspectos teóricos e práticos em Administração está longe de ter um fim, principalmente se for considerado o contexto brasileiro. Isso porque se verifica que as pesquisas acadêmicas da área possuem pouca aproximação e parceiras com empresas atuantes no mercado. Esse 
distanciamento entre aqueles que formulam e testam teorias e aqueles que comandam e agem na prática organizacional só contribui para o acirramento do conflito desses grupos.

É necessário haver um redirecionamento das pesquisas em Administração para demonstrar uma maior preocupação com os avanços que podem ser trazidos para o dia a dia das organizações. Em certa medida, os pesquisadores da área tornam-se reféns do sistema de ensino pelo qual estão submetidos. Por estarem, em sua grande maioria, principalmente no Brasil, dentro das universidades e instituições de ensino e não nas empresas, as preocupações dos estudos são norteadas pelos órgãos regularizados e de apoio ao ensino e pesquisa que possuem seus objetivos próprios e que podem não corresponder aos interesses das empresas públicas e privadas atuantes no mercado.

A fim de demonstrar como os aspectos teóricos da Administração estão orientados para a prática organizacional, foram verificados os trabalhos do EnANPAD 2014. De maneira geral, os trabalhos revelaram certa relação com o universo organizacional, precisando realizar uma maior aproximação das empresas na elaboração dos seus estudos, além do aprimoramento das técnicas de análises estudadas para que essa orientação possa vir a aumentar.

Como limitações, aponta-se que a pesquisa fica restrita à discussão entre os aspectos teóricos e práticos levantados no decorrer do estudo. Ademais, quanto aos resultados obtidos junto aos artigos, denota-se que os mesmos não podem ser generalizáveis aos demais trabalhos publicados em outros eventos e revistas acadêmicas da área. A partir disso, para pesquisas futuras, sugere-se expandir a discussão entre a aproximação de trabalhos acadêmicos no contexto organizacional dentro da área como, por exemplo, aprofundando aspectos da cientificidade da Administração, além da verificação dessa aproximação em outros eventos científicos ou em programas de pós-graduação e periódicos acadêmicos.

\section{REFERÊNCIAS}

ALBACH, H. La Economia de La Empresa como Ciência. Alcalá: Universidad de Alcalá, 1993.

AUDET, M. Le procès des connaissances de l'administration. In: AUDET, M.; MALOUIN, J. (Eds.). La production des connaissances scientifiques de l'administration. Quebec: Université Laval, p.23-56, 1986.

BARBOSA, M. A. C. et al. Nem só de Debates Epistemológicos Vive o Pesquisador em Adminitração: Alguns apontamentos sobre disputas entre paradigmas e campo científico. Cadernos EBAPE.BR, Rio de Janeiro, v. 11, n. 4, a. 9, p. 636-651, 2013. 
BERTERO, C. O.; CALDAS, M. P.; WOOD JR, T. Produção científica em administração de empresas: provocações, insinuações e contribuições para um debate local. Revista de Administração Contemporânea, v. 3, n. 1, p. 147-178, 1999.

BOURDIEU, P. Os usos sociais da ciência: por uma sociologia clínica do campo científico. São Paulo: Ed. Unesp, 2004.

DIJK, N.; PUNCH, M. Useful Knowledge. Management Science as Dialogue and Confrontation, 1993.

DJALÓ, A. B.; PROCOPIUCK, M. Teoria e Prática na Construção do Conhecimento em Administração. Revista Eletrônica de Ciência Administrativa, Campo Largo, v. 9, n. 1, p. 90-103, 2010.

GIL, A. C. Como elaborar projetos de pesquisa. Quinta edição. São Paulo: Editora Atlas S. A., 2010.

HAIR JR., J. F.; BABIN, B.; MONEY, A. H.; SAMOUEL, P. Fundamentos de Métodos de Pesquisa em Administração. Porto Alegre: Bookman, 2005.

JOHANN, E. R.; DUCLÓS, L. C. A Administração como ciência orientada à prática. Revista ADMpg Gestão Estratégica, Ponta Grossa, v. 6, n. 2, p. 17-24, 2013.

KUHN, T. S. A estrutura das revoluções científicas. São Paulo: Perspectiva, 1991.

MARCONI, M. A.; LAKATUS, E. M. Fundamentos da metodologia científica. Sexta edição. São Paulo: Atlas, 2005.

PEREIRA, C. de B.; TOLEDO, G. L.; TOLEDO, L. A. Considerações sobre o Conceito de Marketing: Teoria e Prática Gerencial. Organizações \& Sociedade, v. 16, n. 50, p. 519-543, 2009.

POPPER, K. Conhecimento objetivo: uma abordagem evolucionária. São Paulo: Edusp, 1975.

RODRIGUES, M. S. Epistemologia e Administração: (Um esboço de) reflexões e possibilidades. Perspectivas Contemporâneas, v.8, n. 1, p. 101-124, 2013.

VIZEU, F. Em algum lugar do passado: contribuições da pesquisa histórica para os estudos organizacionais brasileiros. Anais... Encontro Nacional da Associação Nacional de PósGraduação E Pesquisa Em Administração, Rio de Janeiro, 2007.

WEICK, K. E. Agenda setting in organizational behavior: a theory-focused approach. Journal of Management Inquiry, v. 1, n. 3, p. 171-182, 1992. 